\title{
Improved Adaptive Routing for Multihop IEEE 802.15.6 Wireless Body Area Networks
}

\author{
Shariar Imtiaz \\ Ahsanullah University of Science and Technology, Dhaka, Bangladesh \\ E-mail: shariarimtiaz@live.com
}

Md. Mosaddek Khan

University of Dhaka, Dhaka, Bangladesh

E-mail: mosaddek@cse.univdhaka.edu

Dr. Md. Mamun-or-Rashid

University of Dhaka, Dhaka, Bangladesh

E-mail:mamun@cse.univdhaka.edu

Dr. Md. Mustafizur Rahman

University of Dhaka, Dhaka, Bangladesh

E-mail:mustafiz@cse.univdhaka.edu

\begin{abstract}
Wireless Body Area Network has the ability to collect and send data on body measurement to the server through PDA or other device. Nodes (sensors) collect vital signs from the body or environmental factor and check them. In IEEE 802.15.6 routing is discussed as a part of the link layer where multihop is not fully considered. Imp roving network performance, reducing energy consumption, thus extending the network lifetime is the main challenge in BANs. Several studies mention that multihop for BANs helps for achieving network performance, reducing energy consumption and extending network lifetime. One work presents the Adaptive multihop tree-based Routing (AMR) protocol that is extensively evaluated in a real testbed deployment. They use fuzzy logic to combine all metrics they use. Another limitation is that they have used Prim's algorithm which is not a realistic approach. So in this work we have improved their multihop treebased Routing (AMR) protocol using Kruskal's algorithm instead of Prim's algorithm. The time complexity of Kruskal's algorithm is way less than prims's algorithm. We have used network simulator 3 (NS3) to simulate and found that our algorith $m$ is better than AMR if many of nodes.
\end{abstract}

Index Terms - WBAN, BAN, Routing, Multihop, Prim's Algorithm, Kruskal's Algorithm, Fuzzy Logic

\section{Introduction}

Wireless Body Area Network (WBAN) is composed of miniature sensor nodes that are capable of taking vital signs from human body and sending to a central node. A central node can process this data and pass it to the specialists via Internet [1]. In WBAN nodes are capable of sampling, processing vital signs of the body such as blood pressure, temperature, sugar level, location, oxygen level etc. Elderly and handicapped people can be easily monitored by using WBAN. They don't need to stay in hospital beds anymore. So WBAN makes life easy. Other applications of WBAN ranged from video games to military application.

IEEE 802.15.6 talks about BAN's physical and medium access layer [2]. They proposed a star architecture and a small area. Here the nodes are directly connected into a central node. But this star architecture is not enough for WBAN, especially if WBAN is in time varying condition [3]. To provide robustness, star architecture has to be replaced by another algorith $\mathrm{m}$. This algorith $\mathrm{m}$ can be mesh, tree or cluster based algorithm. These mesh, cluster or tree is called multihop architecture. Multihop usage can also reduce power consumption.

WSN is a common term in networking. Several works have been done on it. WSN has several protocols [4]. These protocols are energy-efficient [5]. Though WBAN is specialized from of WSN but these protocols cannot be used in WBAN due to special properties of WBAN. WBAN has some limitations such as low energy, short communication range, irregular traffic etc. But recently several protocols have been proposed for WBAN [6] [7] considering the limitations of WBAN and how to deal with it.

As nodes of WBAN are miniaturized and batterypowered, energy consumption is a major fact in WBAN. 
So with a view to saving energy the physical device, network device, protocols and application should be energy-efficient. Most of the existing protocols which are so-called energy-efficient choose a static optimal path to the sink node and they don't save power in real life. This leads to unbalanced energy distribution among sensor nodes. This unbalanced energy consumption reduces network life time and causes network disconnection [8].

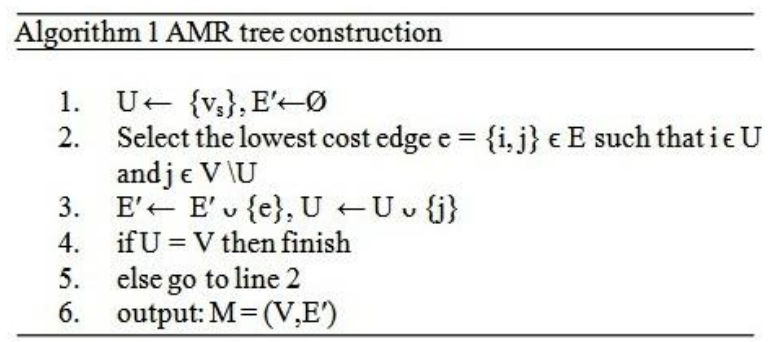

IAMR is more cost-effective than AMR. And almost work the similar way as AMR works. In the next few sections it is discussed how this protocol works. In background studies the working procedure of AMR and its routing metrics; message formats and its details are depicted. In the next section IAMR along with its all routing metrics are discussed. In the simulation it is shown how IAMR is better than AMR.

\section{Background Studies}

\subsection{Network Model and Tree Construction}

If we assume that $\mathrm{v}$ is the Set of vertices which represents the nodes in the network and $E$ is the set of edges which represent network communication topology then WBAN can be modeled as $\mathrm{G}=(\mathrm{V}, \mathrm{E})$, here $G$ is undirected graph . Now an edge $\left(v_{i}, v_{j}\right) \in E$ if and only if $\mathrm{v}_{\mathrm{i}}, \mathrm{v}_{\mathrm{j}}$ are in each other's communication range. The central node is defined as $\mathrm{v}_{\mathrm{s}}$. The resulting multihop routing tree constructed by $A M R, M=\left(V, E^{\prime}\right)$ is a spanning tree, which has all the vertices of $G$ and $E^{\prime} \epsilon$ E, represents the subset of edge that are included in the tree depending on the metric used. This operation follows the prim's approach to construct spanning tree [9] as illustrated Algorithm 1. After adding the central node to the tree, edges are iteratively selected in BFS based on the adopted parent selection metric until all nodes have been added.

In order to communicate with nodes AMR use some massages. They are as follows-

HELLO: this message triggers the discovery process. Upon receiving this message, nodes start the parent selection process.

JOIN: when selecting a parent node, say node b, a node, say node a, sends a JOIN request to the candidate parent $b$. The node $b$ will send back an ACCEPT message to node a.
ACCEPT: parent-child associations are acknowledged in order to ensure child nodes successful association and routing tables are updated.

LEAVE: a node having selected a parent may have to change it due to low residual energy. Nodes will consider the residual energy, and time to die will be estimated. If this time to die is lower than a prefixed threshold (i.e., the energy needed to finish the current round), nodes having a parent without enough residual energy to finish the current round, will send a DEL message (i.e., leave request) and will select as parent any other node with enough residual energy to finish the current round.

DATA: after selecting and associating to a parent, nodes can start sending data packets that include body measurements.

\subsection{Routing Metrics}

Though AMR can adapt many metrics, we use hop count using SPT, RSSI, and battery level. We further use fuzzy logic to combine these three metrics similar to our provided work [10].

Shortest Path Tree (SPT): SPT is a tree based network in which all nodes have the lowest number of hop count to the central node. This metric is directly related to end to end delay. But node overload in relaying node supporting a high number of child nodes may cause data loss and network connectivity failures.

Received Signal Strength Indicator (RSSI): The use of the high RSSI link ensures the correct communication between nodes in the network.

Residual energy (Battery): In BAN it is important to increase network and battery lifetime. Energy consumption must be balanced across the network to ensure maximu m network lifetime.

Fuzzy Logic: Fuzzy logic is a decisive approach that enables the efficient combination of different parameter that can be used as a single metric [11]. It is widely used in machine control. The term itself inspires certain skepticism, sounding equivalent to "half-baked logic" or "bogus logic", but the "fuzzy" part does not refer to a lack of rigor in the method, rather to the fact that the logic involved can deal with concepts that cannot be expressed as "true" or "false" but rather as "partially true". Although genetic algorithms and neural networks can perform just as well as fuzzy logic in many cases, fuzzy logic has the advantage that the solution to the problem can be cast in terms that human operators can understand, so that their experience can be used in the design of the controller. This makes it easier to mechanize tasks that are already successfully performed by humans. 
Table 1: Fuzzy rule set

\begin{tabular}{|c|c|c|c|c|}
\hline \multirow{2}{*}{$\begin{array}{c}\text { Number of } \\
\text { Hops }\end{array}$} & \multicolumn{3}{|c|}{ Residual } & \multirow{2}{*}{ RSSI } \\
\cline { 2 - 4 } & Low & Medium & High & \\
\hline near & bad & bad & median & poor \\
\hline near & bad & median & good & average \\
\hline near & median & good & good & good \\
\hline medium & bad & bad & bad & poor \\
\hline medium & bad & median & median & average \\
\hline medium & median & median & good & good \\
\hline far & bad & bad & bad & poor \\
\hline far & bad & median & median & average \\
\hline far & median & median & median & good \\
\hline
\end{tabular}

A typical FLS, widely used in fuzzy logic controllers is composed of fuzzifier, fuzzy rules, inference engine and defuzzifier. The operation of an FLS can be summarized as follows: crisp data are fuzzified and converted into fuzzy values. These fuzzy values are evaluated by the inference engine by considering a set of rules that relates the input and output variables. The output value obtained in the previous step is then defuzzified, providing a numerical value that can be used as a metric by the external system. The computational requirements of an FLS are usually lower than conventional mathematical operation such as arith metic operations [12]. The variables considered for this work is as follows -

Number of hops: it represents that how many times a packet does have to be transmitted to another node to reach the destination node.

Residual energy: since nodes are using battery so the parameter must be considered in order to save energy and increase lifetime.

RSSI: nodes with high RSSI will prefer because the quality of receiving signal is important to ensure correct data reception.

Input parameters are characterized into a set of linguistic values: Number of hops $\subset$ \{near, medium, far\}, Residual Energy $\subset\{$ low, medium, high\}, RSSIC \{poor, average, good\}, and Output $\subset\{$ bad, median, good\}. Linguistic input values are related to output values following the rules defined in Table 1, which represents rules such as: IF Number of hops is medium AND Residual energy is high AND RSSI is good THEN Output is good.

An illustrative example of the membership functions for the input and output parameters used in our FLS is given in Fig 1. For example, considering the number of hops, labell corresponds to near, label2 to medium and label3 too far. The values X0to X4 have been adjusted according to each input variable, for example, for residual energy, $\mathrm{X} 0=0 \%, \mathrm{X} 1=25 \%, \mathrm{X} 2=50 \%, \mathrm{X} 3=$ $75 \%$, and $X 4=100 \%$, considering $100 \%$ when node batteries are fully charged.

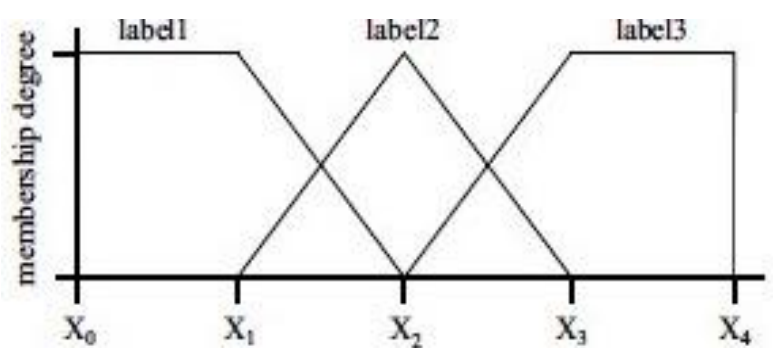

Fig. 1: Membership function example used to define input and out put in fuzzy set

\section{Proposed Protocol}

To construct routing tree we assumed an undirected graph $\mathrm{G}=(\mathrm{V}, \mathrm{E})$ where $\mathrm{V}$ represents the number of nodes and $E$ represents the communication topology. Here everything is same as our base algorithm on which we are working on. But we choose Kruskal's algorithm instead of prim's algorithm to generate the minimum spanning tree. Let assume a set $\mathrm{A}$ which start as an empty set and select at every stage the shortest edge that has not been chosen or rejected, regardless of where this edge is situated in the graph, as illustrated in Algorithm 2. After adding the central node to the tree edges are iteratively selected in BFS approach. The message format will be same as AMR.

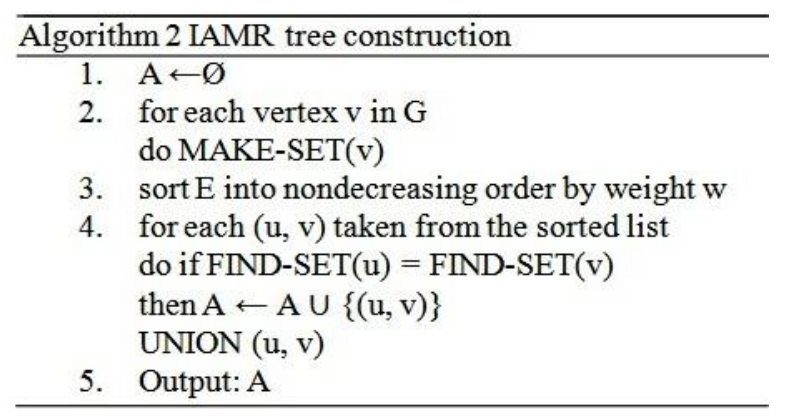

The operation of AMR can be divided into two phases, considering nodes inside and outside the sink coverage. First, the sink node broadcasts a HELLO message, and nodes receiving this message send a JOIN message to the sink in order to associate themselves. The sink node will acknowledge these nodes by sending (unicast) an ACCEPT message. Upon receiving the ACCEPT message, nodes update their routing tables and broadcast a HELLO message. Nodes outside the sink coverage will eventually receive a HELLO message and wait for time $t_{\text {wait }}$ to receive HELLO messages from other neighbor nodes. After $t_{w a i t}$, nodes select their parents based on the selected metric. When a parent is selected, a JOIN message is sent, and if no ACCEPT is received from the selected parent, the JOIN message is resent. After receiving the ACCEPT from the selected parent, nodes send a HELLO message and are ready to send/relay DATA packets. In case of parent node $\mathrm{p}$ not having enough residual energy to fin ish the current round, if it has any child $\mathrm{c}$ already associated top, c sends a LEA VE message to $\mathrm{p}$ and selects another parent node with enough residual energy. The operation 
of AMR can be divided into two phases, considering nodes inside and outside the sink coverage. First, the sink node broadcasts a HELLO message, and nodes receiving this message send a JOIN message to the sink in order to associate themselves. The sink node will acknowledge these nodes by sending (unicast) an ACCEPT message. Upon receiving the ACCEPT message, nodes update their routing tables and broadcast a HELLO message. Nodes outside the sink coverage will eventually receive a HELLO message and wait for time $t_{w a i t}$ to receive HELLO messages from other neighbor nodes. After $t_{w a i t}$, nodes select their parents based on the selected metric. When a parent is selected, a JOIN message is sent, and if no ACCEPT is received from the selected parent, the JOIN message is resent. After receiving the ACCEPT from the selected parent, nodes send a HELLO message and are ready to send/relay DATA packets. In case of parent node $\mathrm{p}$ not having enough residual energy to finish the current round, if it has any child $\mathrm{c}$ already associated top, c sends a LEA VE message to $\mathrm{p}$ and selects another parent node with enough residual energy.

\section{Simulation}

Using different approach that is Kruskal's algorithm instead of Prim's algorithm does not effect on other performance. If we look into Fig 2 we can observe that both of the AMR and IAMR constructs the same tree. They both use same metrics that are hop count, RSSI and battery level. Only one thing that makes them different from one another is time complexity.

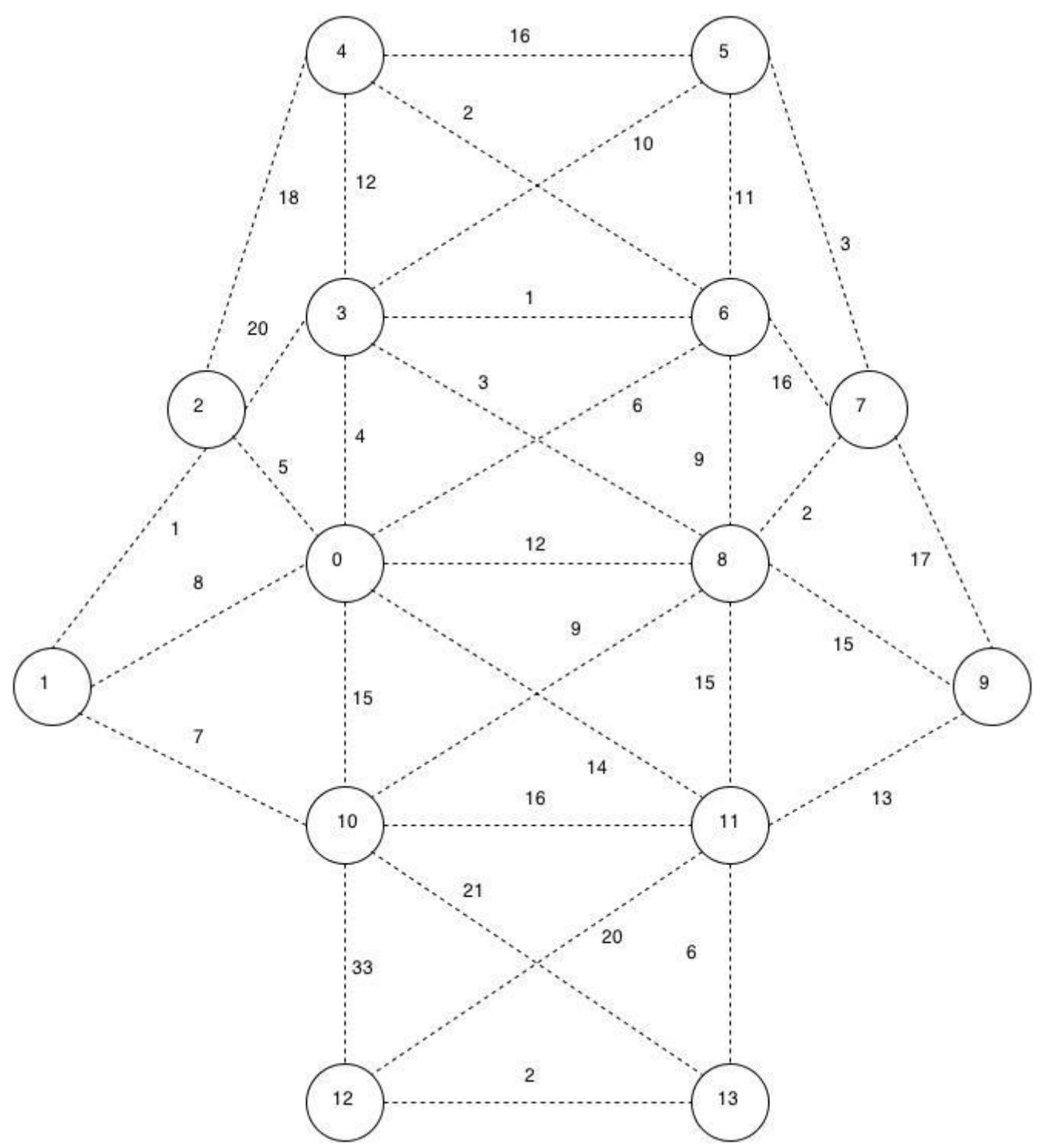

Fig. 2: (a) 

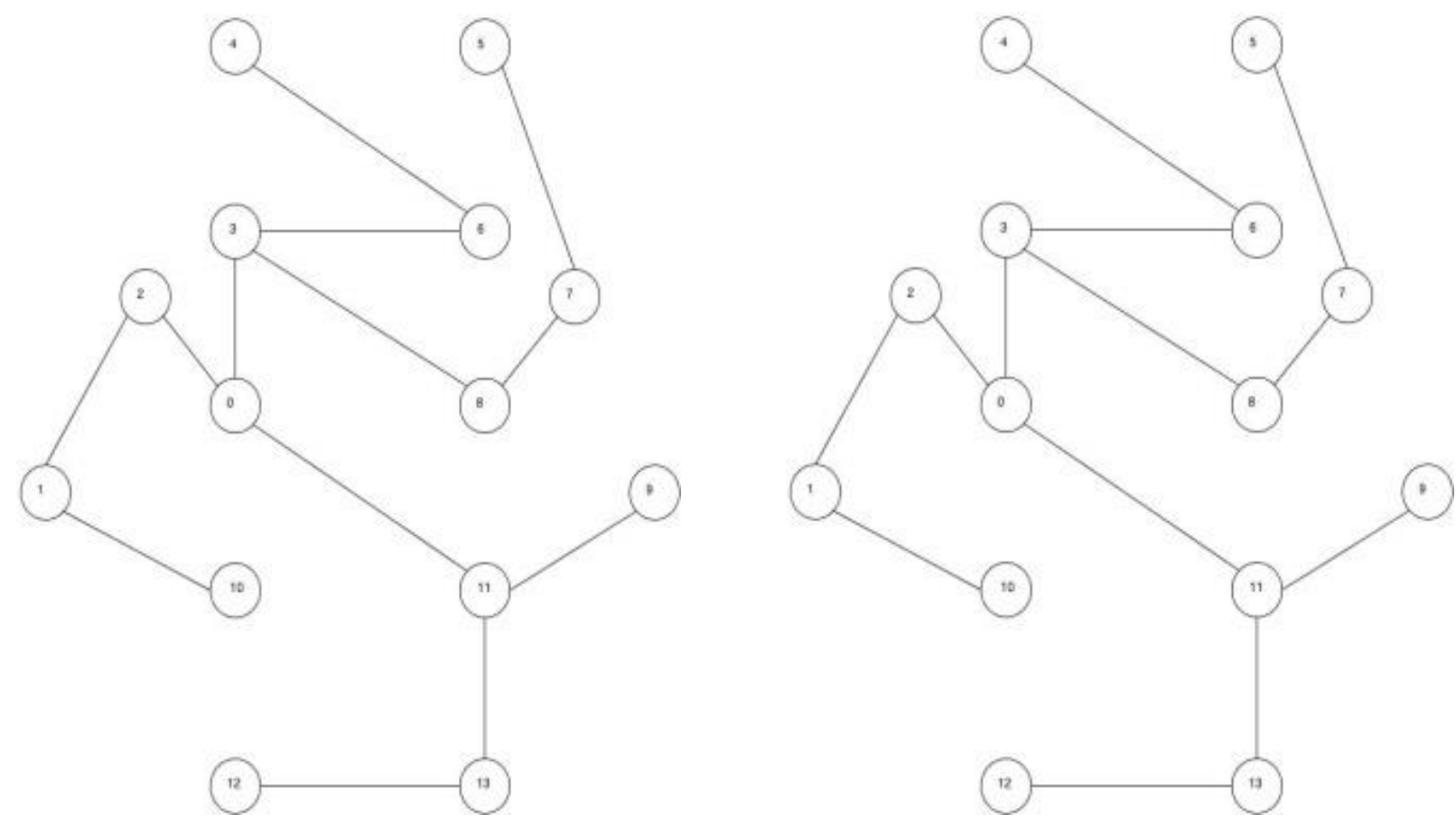

Fig. 2: (a) Present node position in the body (b) Tree using AMR (c) Tree using IAMR

By simulating and using mathematics we can conclude that our proposed algorith $m$ is better than AMR. If we look to Table 2 we can see the difference between the two algorith ms. If we assume that there are more than 100 nodes then we can conclude that AMR takes 960 milliseconds where IAMR takes only 36 milliseconds. If number of nodes is less than 10 then the difference between time complexities is almost zero.

Table 2: Difference between time complexities of two algorithms

\begin{tabular}{|c|c|c|c|c|c|c|}
\hline No. of nodes & 10 & 25 & 50 & 75 & 100 & 125 \\
\hline AMR & 27 & 41 & 150 & 400 & 960 & 1800 \\
\hline IMAR & 27 & 30 & 32 & 34 & 36 & 40 \\
\hline
\end{tabular}

From the table, with an order of 10 the results were almost identical. When the order was increased to 25 , slight differences became apparent and it appeared that Kruskal's algorithm was the fastest. For an order of 50, Prim's algorithm took almost five times the time than during an order of 10 . While Kruskal's had increased by just five milliseconds. The next columns proceed in the same fashion and in the final graph test with an order of 125 vertices it took Prim's algorithm nearly two seconds to complete, while Kruskal's algorithm completed the same task in just 40 milliseconds.

If drawn on a graph, with order in the $\mathrm{x}$-axis and also being the independent variable and $y$-axis as the dependant variable time, the plot would look as follows-

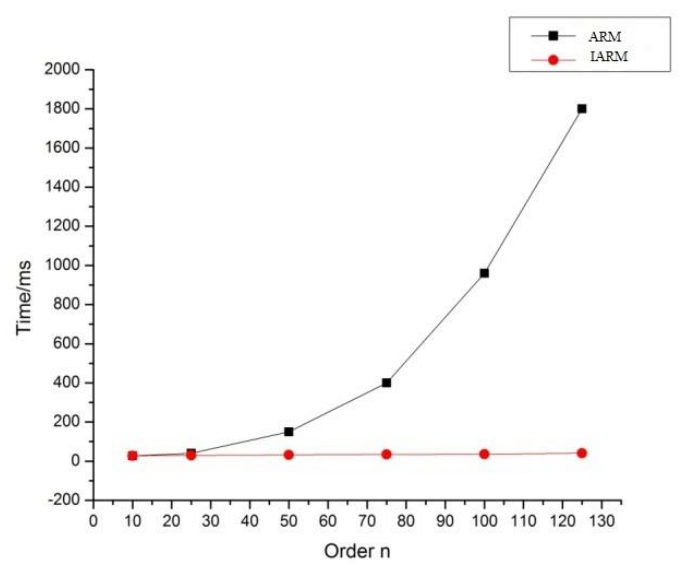

Fig. 3: Difference between time complexity between AMR and IAMR

Fuzzy logic balances the network load better than any other metrics that we proposed. Thus it achieves longer network lifetime. This is shown in Fig 4. This graph is shown in two central locations. In this graph fuzzy logic has better performance. On the other hand battery power metric obtains shortest network lifetime. 


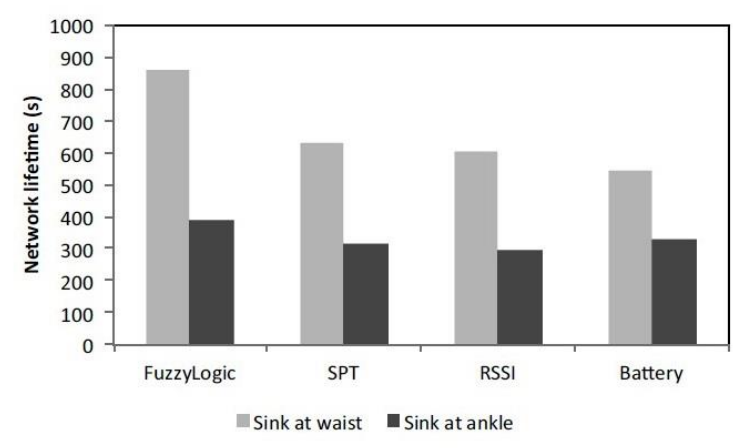

Fig. 4: Network life time for each metric

Fig 5 shows per node Packet Delivery Ratio (PDR). Here also fuzzy logic obtains better overall behavior then the other metrics. Paths that are created by fuzzy logic are more robust. Again battery shows the worst result in the PDR.

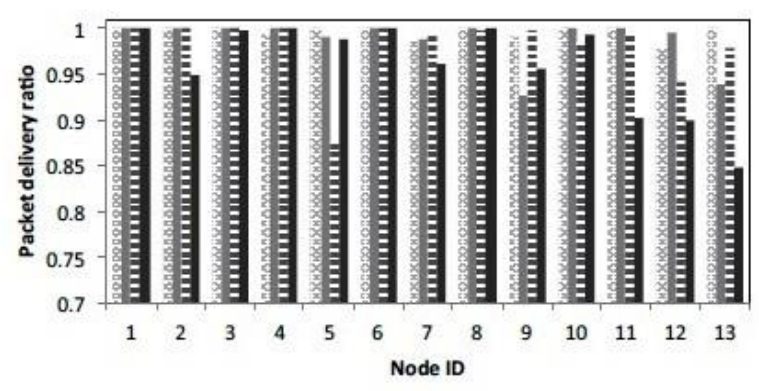

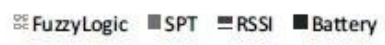

(a) Sink at waist

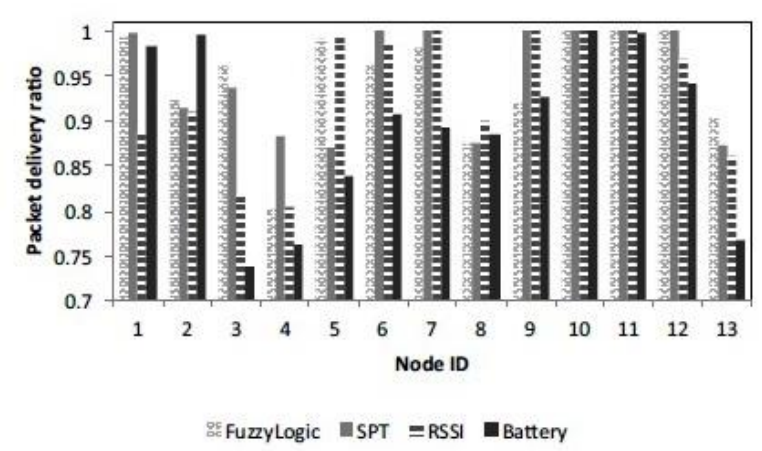

(b) Sink at ankle

Fig. 5: Per node packet delivery ratio (PDR)

The normalized residual energy for all metric is illustrated in Fig 6. Hop count, RSSI actually does nothing to balance out network load. So nodes will drain their battery energy faster than other node cause network partition. But fu zzy logic and battery metric are able to handle network load and balance it. For example when the sink node is located at ankle, most of the network traffic has to be relayed by nodes 10 and 11 , fuzzy logic and battery metrics are able to balance out the network load and keep both nodes with around the same residual energy level, while SPT and RSSI exhaust node 10, making it to rapidly decrease its residual energy level. Routing overhead is directly related to energy consumption.

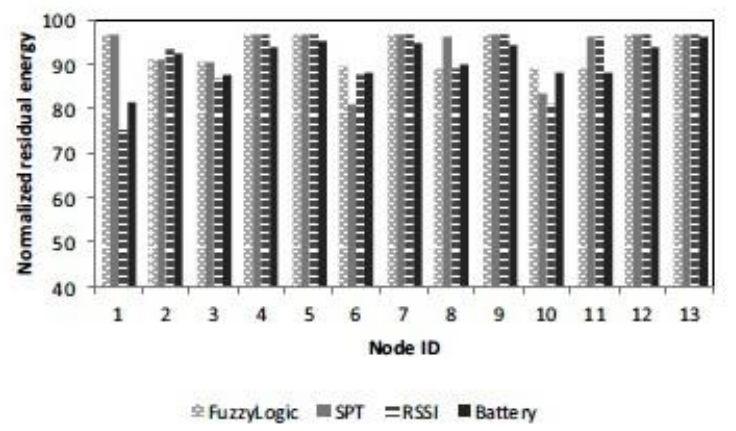

(a) Sink at waist

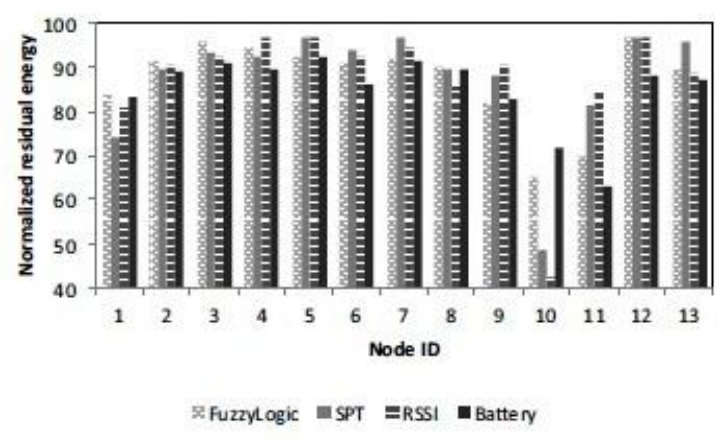

(b) Sink at ankle

Fig. 6: Residual energy distribution of nodes

Now in Fig 7 the average number of transmissions per delivered data packet is shown. SPT obtains the lowest number of transmissions per delivered packet, as packets are forwarded in shortest path. But other metrics may incur longer paths when they provide better overall network performance. For this experiment battery metric performs the worst.

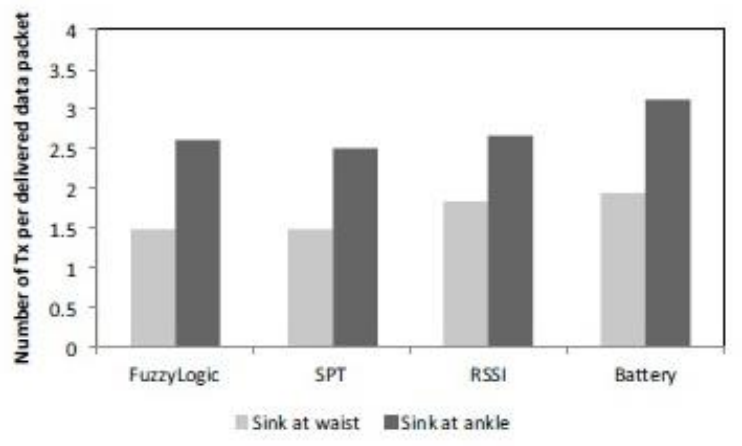

Fig. 7: Number of transmissions (Tx) per delivered data packet

\section{Conclusion}

IEEE 802.15.6 is defined for physical and data link layer for BANs, however, multihop routing in not fully 
discussed. AMR is an adaptive network discovery and routing tree construction protocol for BANs that take into account several metrics in selecting the routing path to the sink or central node. Several routing metrics i.e. number of hops, RSSI, battery level are combined using fuzzy logic. AMR is tested using 14 TelosB based nodes testbed. Like an AMR, IAMR works with same metrics and all the work procedures are same but it has improved in performance. Only one change has been done in IAMR that is using Kruskal's algorithm instead of the prim's algorithm. No effects on used metrics occur because of this change. IAMR just improved time complexity of AMR where we assumed that number of nodes is higher.

\section{References}

[1] B. Latre, B. Braem, I. Moerman, C. Blondia, and P. Demeester, "A survey on wireless body area networks," Wireless Networks, vol. 17, pp. 1-18, 2011.

[2] "IEEE 802.15 WPAN Task Group 6 (TG6) Body Area Networks." [Online]. Available: http://www.ieee802.org/15/pub/TG6.html.

[3] A. Natarajan, B. de Silva, K.-K. Yap, and M. Motani, "To hop or not to hop: Network architecture for body sensor networks," in Proc. of the IEEE SECON, 2009, pp. 1-9.

[4] J. N. Al-Karaki and A. E. Kamal, "Routing techniques in wireless sensor networks: A survey," IEEE Wireless Communications, vol. 11, no. 6, pp. 6-28, 2004.

[5] N. A. Pantazis, S. A. Nikolidakis, and D. D. V., "Energy-efficient routing protocols in wireless sensor networks for health communication systems," in Proc of the International Conference on Pervasive Technologies Related to Assistive Environments (PETRA), 2009.

[6] S. Ullah, H. Higgins, B. Braem, B. Latre, C. Blondia, I. Moerman, S. Saleem, Z. Rahman, and K. Kwak, "A comprehensive survey of wireless body area networks," Medical Systems, pp. 1-30, 2010.

[7] Antonio M.ortiz, NedalAbabnch ,Adaptive Routing for Multihop IEEE 802.15.6 Wireless Body Area Network".

[8] F. Ren, J. Zhang, T. He, C. Lin, and S. Ren, “Ebrp: Energy-balanced routing protocol for data gathering in wireless sensor networks," IEEE Transactions on Parallel and Distributed Systems, no. 99, 2011.

[9] Crossbow Technology Inc, "Telosb datasheet," www.willow.co.uk/TelosB_Datasheet.pdf.Ptive routing generalizations," Bell System Technical Journal, vol. 36, pp. 1389-1401, 1957.
[10] A. M. Ortiz, F. Royo, T. Olivares, and L. OrozcoBarbosa, "Intelligent route discovery for Zig Bee mesh networks," in Proc. of the IEEE WoWMoM, 2011.

[11] J. M. Mendel, "Fuzzy logic systems for engineering: a tutorial," in Proc. of the IEEE, vol. 83, 1995, pp. 345-377.

[12] W. Su and T. C. Bougiouklis, "Data fusion algorithms in cluster-based wireless networks using fuzzy logic theory," in Proc. of the IEEE ICC, 2007.

\section{Authors' Profiles}

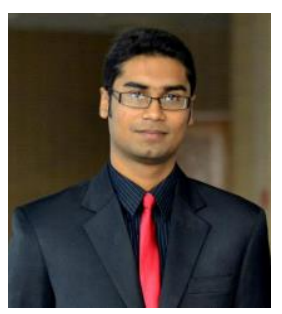

Shariar Imtiaz was born on October 25, 1990. He received his B.Sc. in CSE from Ahsanullah University of Science and Technology, Dhaka, Bangladesh in 2013. Now he is in Banglalink Digital Communication Ltd, Bangladesh as DBA. His research interest includes Wireless Body Area Networks, Wireless Sensor Networks, and Database etc.

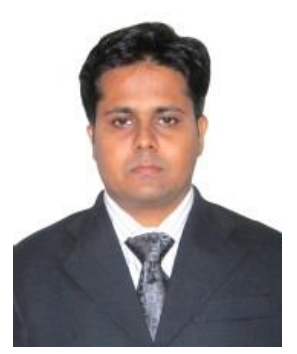

Md. Mosaddek Khan received the B.Sc. (Hons.) in CSE and M.S. in CSE degrees from University of Dhaka, Bangladesh in 2010 and 2012, respectively. He is currently working as a Lecturer at Department of Computer Science and Engineering, University of Dhaka, Bangladesh. His research interests include Mobile Adhoc Networks, Wireless Sensor Networks, Cloud Computing, Wireless Body Area Networks, Robotics, and Data Mining.

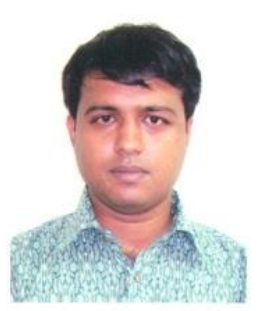

Dr. Md. Mamun-or-Rashid received B.Sc. (Hons.) in Computer Science \& Engineering from University of Dhaka. He received M.Sc.in Computer Science and Engineering from University of Dhaka, Bangladesh from Kyung He University in South Korea. He is currently working as an Associate professor at Department of Computer Science and Engineering, University of Dhaka, Bangladesh. His research interests include Wireless Ad-hoc Networks, Sensor Networks, Wireless Mesh Networks, Future Internet, Multiple Access Control, Congestion control.

Dr. Md. Mustafizur Rahman received M.Sc. in Computer Science and Engineering from University of 


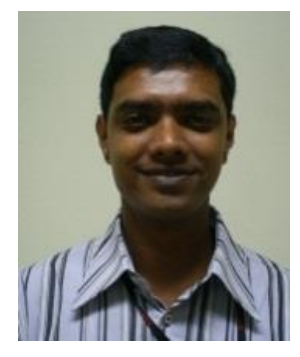

Dhaka, Bangladesh from Kyung He University in South Korea. He is currently working as an Associate professor at Department of Computer Science and Engineering, University of Dhaka, Bangladesh. His research interests include Wireless Ad-hoc Networks, Sensor Networks, Wireless Mesh Networks, Future Internet, Congestion Control, Media Access Control, Wireless Adhoc LAN, and Computer Networks.

How to cite this paper: Shariar Imtiaz, Md. Mosaddek Khan, Md. Mamun-or-Rashid, Md. Mustafizur Rahman,"Improved Adaptive Routing for Multihop IEEE 802.15.6 Wireless Body Area Networks", International Journal of Intelligent Systems and Applications(IJISA), vol.5, no.12, pp.64-71, 2013. DOI: 10.5815/ijisa.2013.12.05 